to set the gas check and warm the gun. These charges gave pressures from previous records of about 11 ton to the square inch. When all was ready, ten rounds, with full charges and projectiles, were fired rapidly, the ten shots being delivered in 19 minutes and 8 seconds.

The gun stood the ordeal without rupture, being the first American high-powered cast steel gun that has endured a full charge firing test of ten rounds. Whether the piece has been injuriously enlarged or strained in the trial, extended experiment alone can show.

\section{TERRACED IRRIGATION PROCESS OF SEWAGE DISPOSAL.}

D.

The problem of sewage disposal cannot as yet be said to be adequately solved. In England, sanitarians propose new methods of treatment continually. Disinfection by chemical treatment, precipitation of the solid matter.by mechanical deposition, or its removal by filtration have all been tried in every conceivable modification. Even electricity has been called in, and th electrolytic treatment is now exciting considerable at tention. It is possible that a wrong conception under lies these attempts. A perfect method seems hardly luting them. If the stream or river ultimately receiv- a fire may be maintained in the base of the chimney to ing the outflow should be in some degree polluted, it increase the draught. The lower ends of the drain will, sooner orlater, become pure again from the effects pipes deliver their flow to a conduit. These ends must of aeration. Simple contact of running water with the be unobstructed and open; and in laying the lines care air tends to purify it from offensive matter. The more should be taken to preserve an even pitch of considerbroken the course of the water, and the thinner the able degree, so as to prevent the possibility of the sheet in which itis exposed to the air, the more effectual formation of traps. The air which the chimney will will be its purification for a given distance or time. draw through the pipes will play an important part in All these principles and methods are utilized in the purifying the drainage water.

arrangement here illustrated. The Waring or subsoil The diluted and filtered and partly aerated sewage irrigation disposal is the basis of the work. The system water collected in the conduit is conducted to a low is represented as applied to providing a sewage ir or a small village or community.

For the sewage farm and disposal works, a piece of the treatment just described, including the three puriground should be chosen that is lower than any of the fying elements of filtration, aeration, and dilution. area included in the sewer or drainage system. The The water finally delivered to the low level conduit field or farm must vary in level; one portion must be at will be comparatively innocuous. It will have been least five feet lower than the other, a greater difference $t$ wice filtered, aerated, diluted, and actied on by vegebeing desirable. This is easily secured by having a tation and humus. Its purification will have proreasonably large piece of ground devoted to the work. gressed in something like a geometrical proportion.

Some kind of surface drainage - a stream or river is best - It will be observed that the above description refers - should be obtainable near at hand.

The sewage is received in a settling tank. In this solid matter is the simplest part of the problem, aud

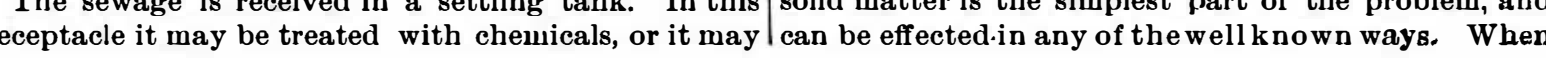

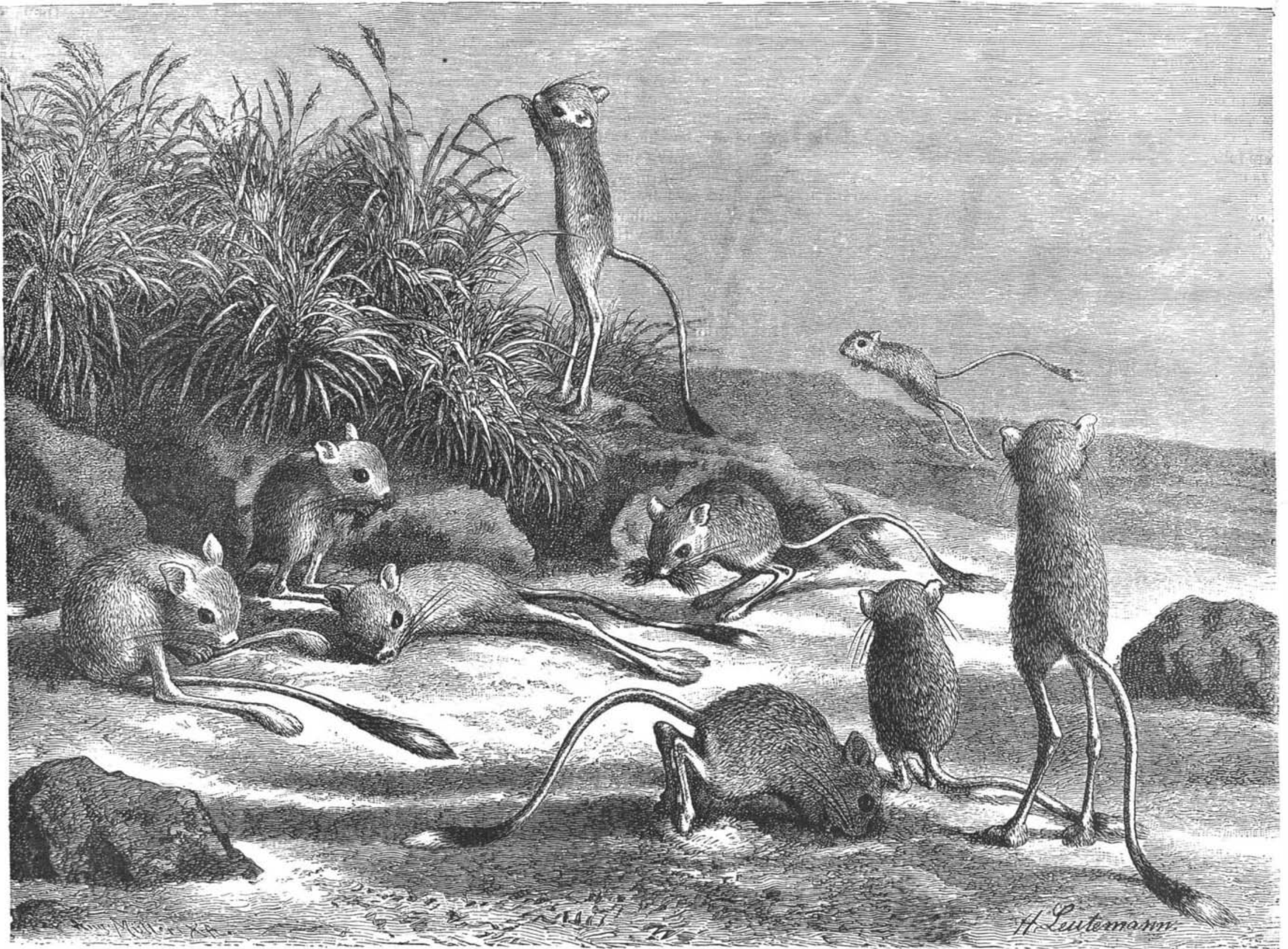

THE JERBOA-[DIPUS IEGYPTIUS LICHTST.]

realizable. Different circumstances make each case individual, and exact individual treatment.

For small systems, the subsoil irrigation method has, up to the present, met with perhaps the greatest success. Under proper conditions, it is quite inoffensive, and can dispose of large quantities of fluid. Its general principle involves the sudden and periodical delivery of the more liquid portions of sewage over a large area of ground about eighteen inches beneath the surface. The liquid matter is disposed of in three ways. A part is absorbed by the roots of the vegetation covering the soil. This portion naturally varies in amount, and in summer is far greater than in winter. A second part evaporates, after penetrating the overlying soil. A third part sinks into the subsoil.

To make the system work well, a piece of ground not too depressed must be chosen, in order that this drainage of the third portion, as specified above, may be ef fectual. If the soil is saturated with natural moisture it will not answer as a filter bed. In such a case, subsoil drainage pipes must be put in below the irrigation system. The water collected by the subsoil lines will be more or less purified by the downward filtration. It will be diluted by natural water so as to be less offengive, and in many cases it can be delivered to the natural overground water courses without perceptibly pol- receive them before entering. If in an isolated local- the aqueous portions of sewage are disposed of, nine ity, the natural precipitation may suffice, the chamber tenths of the problem is solved. containing the tank being properly ventilated. The principal object of chemicals would be to deodorize it. From the settling tank it overflows into the siphoning tank. From this it is periodically discharged by a siphon of any of the well known types. The liquid matter runs into the sub-surface perforated irrigation pipes, and is distributed through the soil.

Where a systematic sewage disposal is the whole function of the area, plants can be selected for cultivation upon the sewage bed that have the greatest power of assiwilating water. Red clover is a good instance, crops might exercise a rood effect, even in winter. attempt should be made to obtain a paying result. The disposal works should be treated as a subject of expense, not of profit.

Three to five or more feet under the sub-surface pipes, a set of regular subsoil drainage pipes are placed. These are represented in the illustration as crossing the others at right angles, though the relative disposihigher ends of these pipes are connected to a high higher ends of these pipes are connected to a high
chimney. This maintains a contiunal aeration of the surfor pipes and water flowing through them. If necessary, in the European-African continent. The result of this

\section{THE JERBOA}

In the diluvial strata of clay at Thiede, near Wolfenbuttel and Westeregeln in the peat district of Magdeburg. Nehring found many fossil remains of the jerboa among other rodents of the plain, and also, as comparison with modern skeletons proves, of a kind of jumping rabbit which is identical with the Alactaga jaculus $B r d t$., still found in the steppes of southwest Siberia and Central Asia. This proves that in the so-called post-glacial period the North German plain, as farback as the mountains of Central Germany, presented the semes, and had a Continental elimate : that is, a hotter summer and a colder winter than at the present time. But it is not probable that the coast of the European continent then had its present form, to which the North German plain owes its moist and mild climate. Europe, especially the western part, must then have been connected with the northern part of Africa, forming a compact 